\title{
Experiencias en relación con la Violencia de Género de la población que consulta en Atención Primaria
}

\author{
Inmaculada González Lozoyaa, Ana Serrano Martíneza , Nuria García Sáncheza, \\ María del Campo Giméneza , Beatriz Moreno Ruiz ${ }^{b}$, Ana Belén González Lozoyac, \\ Julio Montoya Fernández ${ }^{\mathrm{d}}$, Juan Manuel Téllez Lapeira ${ }^{\mathrm{e}}$, Francisco Escobar Rabadán ${ }^{\mathrm{d}}$
}

\begin{abstract}
a Estudiante de la Facultad de Medicina de Albacete.

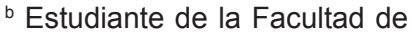
Psicología de Valencia.

c Diplomada en Gestión y Administración Pública.

d Médico de Familia, Centro de Salud Universitario Zona IV de Albacete, Profesor Asociado, Facultad de Medicina de Albacete.
\end{abstract}

e Coordinador del Centro de Salud Zona VB, Profesor Asociado, Facultad de Medicina de Albacete.

Correspondencia: Francisco Escobar Rabadán, Centro de Salud Universitario Zona IV, c/ Seminario $n^{\circ} 4$, 02006 - Albacete, España. Telf.: 967510094 , correo electrónico: fjescobarr@sescam.jccm.es.

Recibido el 30 de marzo de 2010.

Aceptado para su publicación el 2 de junio de 2010.

\section{RESUMEN}

Objetivo. Conocer la prevalencia de violencia de género padecida por la población consultante en Atención Primaria y su relación con factores sociodemográficos y otras experiencias personales.

Diseño. Estudio observacional, descriptivo, transversal.

Emplazamiento. Centros de Salud urbanos.

Participantes. Pacientes de 18 o más años que acuden a consulta de Atención Primaria.

Mediciones principales. Se utilizó un cuestionario diseñado ad hoc, que incluía preguntas relativas a las experiencias vividas en relación con la violencia de género y en el ámbito doméstico, junto a la versión corta del Woman Abuse Screening Tool (WAST). También recogía edad, sexo, nivel de estudios y estado civil, así como conocimientos y actitudes con respecto a este tema. Resultados. Respondieron al cuestionario 673 personas. De las 662 personas que respondieron a la pregunta sobre si ha sufrido algún tipo de violencia en sus relaciones sentimentales, 76 dieron una respuesta afirmativa (11,48\%; IC 95\%: 8,98-13,98\%). Esta respuesta era más probable encontrarla en viudos y separados $(p<0,00001)$, mientras que entre los más jóvenes predominaba el no haber sufrido violencia $(p=0,01)$, sin diferencias para otras variables sociodemográficas. Mediante regresión logística, la edad fue la única variable asociada independiente con haber sufrido violencia de género (OR: 1,02; IC95\%: 1,005-1,036). Para el test WAST se obtuvieron 560 respuestas, de las que un 12,0 \% (IC95\%: 9,2-14,7\%) fueron positivas. La concordancia entre la respuesta a la pregunta sobre si ha sufrido algún tipo de violencia en sus relaciones sentimentales y el resultado del WAST fue muy baja (Kappa: 0,153; IC 95\%: 0,038$0,243)$. A la pregunta "¿qué ha hecho?" (en caso de violencia en alguna relación sentimental), la respuesta más frecuente es alejarse del agresor. Hasta un $42,1 \%$ de los participantes decía conocer a alguna persona que hubiera padecido violencia de género.

Conclusiones. Existe un contacto frecuente de las personas que acuden a consulta de atención primaria con la violencia de género, siendo considerable el número de personas que afirman haber sufrido maltrato.

Palabras clave. Violencia Doméstica, Atención Primaria.

\section{ABSTRACT}

Experience in gender violence in the general population within the Primary Care setting Objectives. To determine the prevalence of gender violence in the general population in the primary care setting and its association with sociodemographic factors and other personal experience.

Design. Cross-sectional, observational, descriptive study.

Setting. Urban primary care centres.

Participants. Patients aged 18 years or over who attended the Primary Care centre.

Main measurements. An ad hoc designed questionnaire that included questions on experience of domestic violence and the short version of the Woman Abuse Screening Tool (WAST), were used. Sociodemographic variables and knowledge and attitudes towards gender violence were also collected.

Results: A total of 673 persons responded to the questionnaires. Of the 662 subjects who answered the question on whether they had suffered any type of abuse by their partner, 75 answered affirmatively (11.48\%; 95\% Cl: 8.98-13.98\%). This response was more probable in widowed or separated subjects $(p<0.00001)$. Amongst young people there was a predominance of not having suffered abuse $(p=0.01)$. There were no differences regarding the other sociodemographic variables. Logistic regression analysis revealed that age was the only variable that demonstrated an independent association with having suffered gender violence (OR: 1.02; 95\% Cl: $1.005-$ 1.036). A total of 560 responses were obtained in the WAST questionnaire and $12.0 \%$ were positive $(95 \% \mathrm{Cl} ; 9.2-14.7 \%)$. Its concordance with the question on history of domestic violence was very low (Kappa: $0.153 ; 95 \% \mathrm{Cl}: 0.038-0.243$ ). In the case of previous abuse, in answer to the question "What did you do? the most frequent response was to get away from the aggressor. Up to $42.1 \%$ said they knew someone who was a victim of gender violence.

Conclusions. In the primary care setting there is frequent contact with persons suffering gender violence. There are a considerable number of persons who admit to being abused.

Key words. Domestic Violence, Primary Health Care.

Una versión preliminar de este trabajo fue presentada en la XXVII Edición del Congreso Nacional de Estudiantes de Medicina \& V International Edition (CNEM) (Alicante, 21, 22 y 23 de octubre de 2009). 


\section{INTRODUCCIÓN}

La violencia de género se está convirtiendo en uno de los temas más cotidianos en nuestra sociedad. Solo en 2008 murieron 75 mujeres a manos de sus parejas, y aunque en 2009 se ha dado una tendencia a la baja, el número de víctimas (55) sigue siendo alto, teniendo en cuenta además que durante los seis primeros meses de ese año se produjo una media diaria de 380 denuncias al día ${ }^{1}$.

Sin embargo, la población en general la considera una circunstancia lejana a su entorno. No en vano esta lacra social la han venido arrastrando con frecuencia en silencio las mujeres a lo largo de la historia. La violencia por parte de una pareja íntima es un significativo problema de salud pública que tiene consecuencias tanto a corto como a largo plazo sobre la salud física y mental de las mujeres y sus familias. Las mujeres que sufren este tipo de violencia utilizan de forma desproporcionada los servicios sanitarios y realizan más visitas a servicios de urgencia y a centros de atención primaria y salud mental que las mujeres que no la sufren, y tienen una probabilidad dos veces mayor de manifestar una discapacidad, como enfermedades cardiacas o circulatorias, problemas de espalda, dolor crónico, artritis, afectación del sistema nervioso, asma u otro problema respiratorio y depresión u otra enfermedad mental ${ }^{2,3}$.

En población española consultante en atención primaria, las cifras de maltrato a lo largo de la vida oscilan en torno a un tercio de las mujeres ${ }^{4,5,6}$, aunque algún estudio presenta cifras cercanas al $50 \%{ }^{7}$. Estos trabajos se centran en el estudio de la población femenina, indudablemente la más afectada, pero no tenemos información sobre la situación por la que atraviesan los hombres.

El objetivo principal de nuestro estudio es conocer la prevalencia de violencia de género padecida por la población consultante en Atención Primaria en nuestro medio y su relación con factores sociodemográficos y otras experiencias personales.

\section{MATERIAL Y MÉTODO}

Hemos realizado un estudio de tipo observacional, descriptivo y transversal, en el que han participado pacientes de 18 o más años que acudían a consultas de Atención Primaria en los Centros de Salud Zona IV y Zona V de Albacete, durante los días 6 a 15 de julio de 2009. Se trata de centros urbanos en los que se atiende a una población, en su mayoría, de nivel socioeconómico medio-bajo.

El tamaño muestral previsto fue de 683 sujetos (proporción esperada del 20\%, precisión del 3\% y nivel de confianza del 95\%).

Para la recogida de información se utilizó un cuestionario diseñado ad hoc, que incluía preguntas sobre los conocimientos, actitudes y experiencias vividas en relación con la violencia de género y en el ámbito doméstico. Tras una revisión bibliográfica exhaustiva elaboramos un borrador, que fue valorado por los 3 profesores de Atención Primaria implicados en el estudio, para asegurar la relevancia y comprensión de las preguntas, y evaluar cualitativamente su validez de contenido y construcción. A partir de los comentarios y sugerencias aportados se elaboró la versión definitiva. El cuestionario, autoadministrable y anónimo, también recogía variables sociodemográficas: edad, sexo, nivel de estudios y estado civil. Además se contemplaba la detección de posibles experiencias personales de Violencia de Género mediante la versión corta del Woman Abuse Screening Tool (WAST). Se trata de un cuestionario autoadministrable, validado en España, que ha demostrado ser efectivo para identificar, de una forma rápida, aquellas mujeres que puedan estar sufriendo violencia de sus parejas ${ }^{8}$.

Se garantizó el anonimato en las respuestas y la preservación de la intimidad del participante. Las respuestas fueron codificadas y registradas en una base de datos Microsoft Excel, para su posterior análisis con el programa estadístico SPSS 17.0. EI análisis estadístico ha consistido en la descripción de las frecuencias de las distintas variables, seguido por una análisis bivariante en el que se comparó la prevalencia de violencia de género padecida en relación con distintos valores de las variables sociodemográficas, por medio del test de chi cuadrado de Pearson en el caso de las variables cualitativas y los tests $U$ de Mann-Whitney y Kruskal Wallis en el caso de las cuantitativas. Finalmente se realizó un análisis de regresión logística, en el que la variable dependiente considerada fue el haber padecido violencia de género.

\section{RESULTADOS}

Se cumplimentaron 704 encuestas en los Centros de Salud Zona IV (335) y Zona V (369) de Albacete, de las que fueron consideradas válidas 673 , tras descartar aquellas en las que faltaban las respuestas en más del $40 \%$ del cuestionario. La muestra estaba constituida por un $68 \%$ de mujeres y el rango de edad fue de 18-86 años; un 63,6\% tenían una edad de 45 años o menos, siendo la media de edad de 41,7 años (DE: 15,3). En la tabla 1 se describen las características sociodemográficas de los sujetos que participaron en el estudio, así como las respuestas a la pregunta sobre si ha sufrido algún tipo de violencia en sus relaciones sentimentales. De las 662 personas que contestaron a esta pregunta, 76 lo hicieron de forma afirmativa (11,5\%; IC 95\%: 9,0-14,0\%). Esta respuesta fue más probable encontrarla en viudos y separados $(p<0,00001)$, mientras que el no haber sufrido violencia predominaba entre los más jóvenes (mediana 40 años 


\begin{tabular}{|c|c|c|c|}
\hline & $\begin{array}{c}\mathrm{Si} \\
\text { (\% de los que responden) }\end{array}$ & No consta & Total (\%) \\
\hline \multicolumn{4}{|l|}{ Edad } \\
\hline$<31$ & $12(7,2)$ & 3 & $170(25,3)$ \\
\hline $31-39$ & $6(10,5)$ & 1 & $154(22,9)$ \\
\hline $40-50$ & $23(13,2)$ & 4 & $178(26,4)$ \\
\hline$>50$ & $25(14,9)$ & 3 & $171(25,4)$ \\
\hline \multicolumn{4}{|l|}{ Sexo } \\
\hline Mujer & $55(12,2)$ & 5 & $455(67,6)$ \\
\hline Hombre & $21(9,9)$ & 6 & $218(32,4)$ \\
\hline \multicolumn{4}{|l|}{ Estado civil } \\
\hline Soltero & $18(9,9)$ & 3 & $184(27,3)$ \\
\hline casado/pareja & $39(9,4)$ & 7 & $421(62,6)$ \\
\hline Separado & $13(33,3)^{*}$ & 0 & $39(5,8)$ \\
\hline Viudo & $6(21,4)^{\star}$ & 1 & $29(4,3)$ \\
\hline \multicolumn{4}{|l|}{ Nivel de estudios } \\
\hline Sin estudios & $2(13,3)$ & 0 & $15(2,2)$ \\
\hline Lee y escribe & $8(22,2)$ & 0 & $36(5,3)$ \\
\hline Primarios & $12(8,2)$ & 2 & $149(22,1)$ \\
\hline Secundarios & $18(10,4)$ & 4 & $177(26,3)$ \\
\hline Titulado medio/superior & $36(12,4)$ & 5 & $296(44,0)$ \\
\hline \multicolumn{4}{|l|}{ Centro de salud } \\
\hline Zona IV & $36(11,3)$ & 4 & $323(48,0)$ \\
\hline Zona V & $40(11,7)$ & 7 & $350(52,0)$ \\
\hline
\end{tabular}

Tabla 1. Respuestas a la pregunta sobre si ha sufrido algún tipo de violencia en alguna de sus relaciones sentimentales, en función de las diferentes variables sociodemográficas analizadas. *Viudos + separados vs solteros y casados: $p<0,00001$.

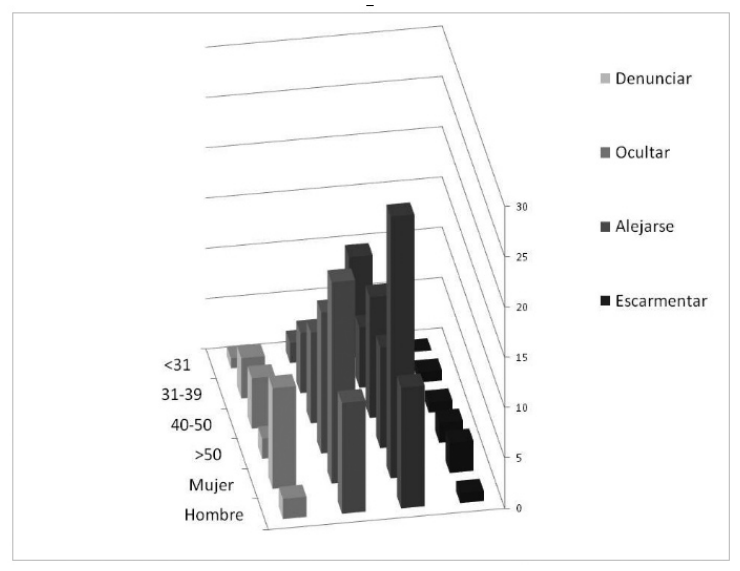

Figura 1. Número de personas que elige cada una de las opciones de respuesta a la pregunta "¿qué ha hecho en caso de haber sufrido algún tipo de violencia en alguna de sus relaciones sentimentales?”, en función de su edad y sexo. Las medianas de edad (RI) son, respectivamente, para quienes han denunciado, tratado de ocultar, procurado alejarse o intentado un escarmiento, de 40 (34-46), 48 (39-69), $44(29,75-52,5)$ y $54,5(35,5-68,25)(p=0,04)$.

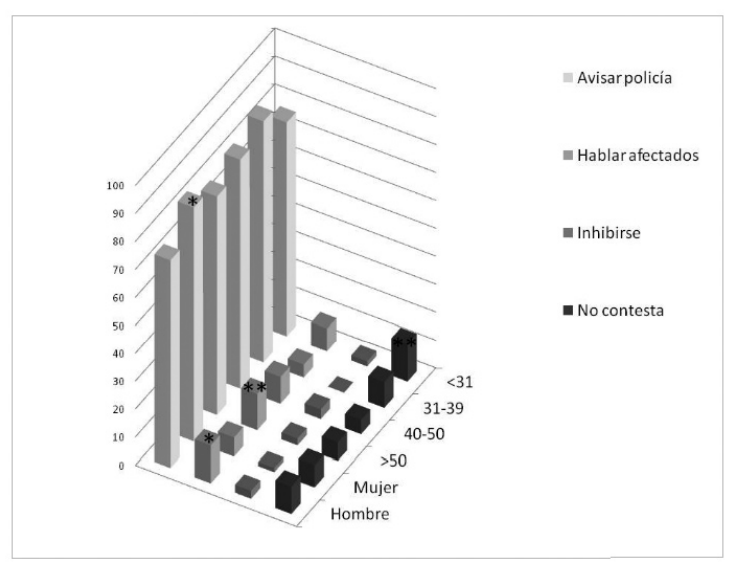

Figura 2. Porcentaje de personas que elige cada una de las opciones planteadas como actuación en caso de que fuera testigo de un episodio de violencia de género, en función de su edad y sexo. * $p=0,007,{ }^{* *} p$ $=0,05$. 
y rango intercuartiles (RI) $30-50$ vs mediana 46 y RI 33-56 para los que la habían sufrido; $p=0,01$ ). No existían diferencias significativas para las demás variables sociodemográficas. $A$ través de un análisis de regresión logística solo se demostró una asociación independiente con el hecho de haber sufrido violencia de género con la edad (OR: 1,02; IC95\%: 1,005-1,036).

A la pregunta "¿qué ha hecho?" (en caso de haber sufrido algún tipo de violencia en alguna de sus relaciones sentimentales), la respuesta más frecuente fue alejarse del agresor, seguida por tratar de ocultar el maltrato, denunciar, y, excepcionalmente, haber intentado escarmentar al agresor. En la figura 1 se muestra el número de personas que elegía cada una de las opciones en función de su edad y sexo. Las medianas de edad y RI son, respectivamente, para quienes han denunciado, tratado de ocultar, procurar alejarse o intentar un escarmiento, de 40 años (34-46), 48 (39-69), $44(29,75-52,5)$ y $54,5(35,5-68,25)(p=0,04)$. No existían diferencias por sexo, estado civil, nivel de estudios, por centro de salud ni por resultado del WAST.

Para el test WAST se obtuvieron 560 respuestas (éstas venían condicionadas por el hecho de tener pareja), 105 con puntuación de 1 y 67 con puntuación 2, de forma que un 12,0 \% (IC95\%: 9,2-14,7\%) fueron consideradas positivas. No se han encontrado diferencias significativas en la distribución de pacientes con WAST positivos o negativos en función de edad, sexo, nivel de estudios, el estado civil ni centro de salud. La concordancia entre la respuesta a la pregunta sobre si ha sufrido algún tipo de violencia en sus relaciones sentimentales y el resultado del WAST es muy baja (Kappa: 0,153; IC 95\%: 0,038-0,243).

Un total de $140(20,8 \%)$ sujetos que participaron en el estudio decían haber presenciado episodios de violencia de género, sin diferencias por sexo, estado civil ni por nivel de estudios. Sí que se encontró diferencia en función del centro de salud a que pertenecían, habiendo presenciado episodios de violencia un $17,3 \%$ de los individuos del Zona IV frente a un $24,0 \%$ de los del Zona $V(p=0,03)$, y de la edad, siendo menos probable para los menores de 30 años $(p=0,05)$. Un $34,3 \%$ de los pacientes con un resultado positivo del test WAST habían presenciado episodios de violencia, frente a un $18,1 \%$ de los que tenían un resultado negativo $(p=0,002)$. Así mismo, el $63,3 \%$ de los encuestados respondía que alguna vez le han contando un episodio de estas características. No había diferencias para el hecho de que le hubieran contado algún episodio de violencia de género con relación a las variables sociodemográficas y el WAST.

Un $24,7 \%$ de los encuestados conocía a alguna persona cercana (se especificaba si familiar, amigo, compañero de estudios, vecino, etc.) que estuviera sufriendo violencia de género y un $38,4 \%$ que la hubiera padecido en algún momento de su vida. No existían diferencias con relación a esta última circunstancia en relación con edad, sexo o estado civil, aunque sí por nivel de estudios (era más probable que no conocieran a personas en esta situación aquellos sin estudios o con primarios incompletos; $p=0,04$ ), y por centro de salud (más probable que conocieran personas en esta situación los pacientes del Zona $V ; p=0,008)$. También era más probable para aquellos con un resultado positivo del WAST $(55,2 \%$ vs $44,8 \% ; p=0,005)$. Si la pregunta se refería a alguien no tan cercano, los porcentajes son respectivamente 34,6 y $42,1 \%$, sin que existieran para éstos diferencias por edad y sexo, estado civil, nivel de estudios, centro de salud ni resultado de WAST.

Al plantear al encuestado la situación de ser testigo de un episodio de violencia de género, para comprobar cuál sería su posible reacción, hay que destacar que un $80,4 \%$ avisaría a la policía, aunque algunos de ellos apuntaban que solo lo harían en caso de que hubieran observado comportamientos violentos en esta pareja en reiteradas ocasiones. Un 8,9\% trataría de hablar con los afectados para aclarar la situación, y solo un $1,9 \%$ no llevaría a cabo ningún tipo de actuación. El $9 \%$ no sabe o no contesta qué haría ante esta hipotética situación. Como podemos observar en la figura 2, existían diferencias por edad y sexo. No se encontraron diferencias con relación al estado civil, el nivel de estudios, centro de salud o resultado del WAST.

Se planteó finalmente la posibilidad de que alguien muy cercano al encuestado fuera la víctima del maltrato, o por el contrario el agresor, preguntando cuál sería su actitud. En el primer caso, la respuesta más elegida fue "incitar a la víctima a denunciar" $(81,6 \%)$, seguida por "intentar hablar con la víctima para ayudar" (48,0\%). Muchos de ellos afirmaban que primero hablarían con la víctima y, si así no se solucionase la situación, pasarían a incitarle a la denuncia. En cuanto a la opción de intentar escarmentar al agresor, solo el $11,1 \%$ se decantaba por ella, si tuviera oportunidad. Por último, un 1,8\% tratarían simplemente de evitar el tema.

La posible actuación del encuestado, si el maltratador resultara ser alguien cercano al mismo, se orientaba mayoritariamente hacia la denuncia $(82,2 \%)$, mientras que un $29,1 \%$ intentaría alejarse de él; un 4,1\% no tomarían ningún tipo de medida e intentaría no hablar del tema, un 2,2\% ocultaría la situación y otro $2,2 \%$ trataría de hablar con el maltratador para intentar ayudarle.

\section{DISCUSIÓN}

Existe un contacto frecuente de nuestros pacientes con la violencia de género, y un número considerable de ellos afirma haberla padecido. Así, un $11,5 \%$ 
de las personas que contestaron a la pregunta sobre si había sufrido algún tipo de violencia en sus relaciones sentimentales dieron una respuesta afirmativa. Este porcentaje es Ilamativamente coincidente con el $12,0 \%$ de respuestas positivas al test WAST. Sin embargo, la concordancia entre ambas cuestiones era muy baja. Entendemos que la explicación a esta aparente contradicción hay que buscarla en la limitada eficacia diagnóstica de ambos procedimientos, por lo que con seguridad estamos infravalorando la magnitud del problema.

Como ya hemos señalado, alrededor de un tercio de las mujeres han sufrido en nuestro país violencia en sus relaciones de pareja a lo largo de la vida. En un estudio realizado por Ruiz-Pérez et al $^{9}$ en mujeres de 18 a 65 años, un $32 \%$ se había encontrado en estas situaciones, con un $14 \%$ que refieren abuso psicológico solamente, $7 \%$ abuso físico y psicológico, pero no sexual, y $6 \%$ los tres tipos de abuso.

La violencia hacia la mujer en el ámbito doméstico frecuentemente queda oculta y es prolongada. La propuesta de investigación rutinaria, con el fin de realizar una detección temprana de casos, ha sido controvertida. A los clínicos por lo común les preocupa que puedan molestar a las mujeres al preguntarles acerca de la posible existencia de violencia en el ámbito doméstico. En un estudio realizado por Boyle y Jones ${ }^{10}$ solo un $8,4 \%$ de las mujeres encontraban inaceptable que les preguntaran sobre este tema los profesionales de la salud. Era más probable que estas mujeres hubieran sufrido abuso en el año previo (OR: 4,5; IC95\%: 1,8-11,4), pero no a lo largo de su vida (OR: 0,9; IC95\%: 0,5-1,9). Por tanto, la investigación de la posible existencia de violencia doméstica por parte del personal sanitario es aceptable, pues, para la mayoría de las mujeres. La aceptabilidad es especialmente elevada para mujeres que no han sufrido abuso en el último año y para las que acuden a clínicas prenatales.

Los temores de los médicos a inmiscuirse en la vida de sus pacientes quedan totalmente refutados a la luz de los trabajos de autores como García Torrecillas ${ }^{11}$ : más del $95 \%$ de las mujeres verían de buen grado que su médico de familia les preguntara acerca de sus relaciones familiares y/o de pareja.

Richardson et $\mathrm{al}^{12}$ entrevistaron a 1.207 mujeres mayores de 15 años que acudían a centros de atención primaria en el este de Londres, por medio de un cuestionario autoadministrado. Encontraron una elevada prevalencia de haber sufrido violencia física por parte de su pareja a lo largo de su vida $(41 \%)$, mientras que un $17 \%$ la habían sufrido en el último año. Solo un $17 \%$ de las mujeres que admitían haber sufrido violencia lo tenían registrado en su historia clínica. Un $20 \%$ de las mujeres no estaban de acuerdo con que se hiciera cribado de violencia doméstica en la consulta. Estos autores ${ }^{13}$ subrayan que, a pesar de que la violencia sexual contra la mujer es común, son pocas las que comunican estas experiencias a sus médicos.

En nuestro estudio la participación ha venido condicionada en gran parte por las circunstancias de espera de los pacientes en cada centro de salud. No obstante, tenemos que admitir que existió una moderada colaboración, que habría que atribuir al hecho de tratarse de un tema incómodo para muchas personas. El análisis de las características sociodemográficas de los participantes ha demostrado un notable sesgo en función del nivel educativo, ya que alrededor de dos tercios de los mismos tienen estudios secundarios o superiores, patrón que sin duda no es el de la población de estas zonas de salud.

Se ha planteado que la asociación entre mala salud percibida y maltrato, así como con probable depresión, podría ser útil para definir a la población en la que se debe sospechar el mismo. De hecho, la depresión se considera entre los indicadores más relacionados ${ }^{14}$. Sin embargo, aunque depresión, ansiedad y somatización se asocian con el maltrato, presentan poco valor predictivo para detectarlo.

Hegarty et $\mathrm{al}^{15}$ encontraron que las mujeres que referían dos o más síntomas físicos en el último mes era más probable que hubieran sufrido experiencias de abuso por parte de un compañero sentimental en los últimos 12 meses. Para la presencia de 3 a 5 síntomas, la OR era de 2,3 (IC 95\%: 1,6 a 3,5), mientras que para 6 a 15 síntomas pasaba a ser de 3,5 (IC 95\%: 2,2 a 5,5). En el análisis multivariante se mostraban indicadores de abuso por parte de la pareja (excluyendo la fuerte asociación con depresión), que incluían características socio-demográficas (edad, separada o divorciada, bajo nivel educativo, bajo nivel de ingresos, no tener seguro privado) y síntomas físicos (diarrea, fatigabilidad, dolor torácico). Por tanto, existe una combinación de características sociodemográficas, cuestiones de salud mental y un creciente número de síntomas físicos y psicosociales comunes que deberían alertar a los clínicos sobre la posibilidad de la existencia de abuso por parte de un compañero íntimo. Sohal et $\mathrm{al}^{16}$ han propuesto el uso de 4 sencillas preguntas que tienen que ver con humillación, miedo, violación y haber sido golpeada. Si un clínico plantea estas cuatro preguntas y su paciente puntúa 1 o más, en un $81 \%$ de los casos se correspondería con mujeres afectadas por violencia de su compañero íntimo.

Cuando planteamos la pregunta "¿qué ha hecho en caso de haber sufrido algún tipo de violencia en alguna de sus relaciones sentimentales?", la respuesta más frecuente fue alejarse del agresor, seguido por tratar de ocultar el maltrato, denunciar, $y$, excepcionalmente, haber intentado escarmentar al agresor. En un reciente trabajo, Muñoz Cobos et $a^{17}$ recogen relatos biográficos de mujeres que 
sufren maltrato. Éstas describen la situación como un estado específico ("estar dentro") mediante la metáfora del "torbellino", caracterizado por ceguera, aislamiento, indefensión, sufrimiento, destino, dependencia, fantasías de amor, protección y cambio, anulación, y círculos viciosos. Las mujeres son conscientes del aguante, y reconocen la importancia de los valores inculcados en la justificación del mismo, con un fuerte peso del ideal de familia. En cuanto a la salida de la situación, se plantea una forma activa y otra pasiva. La forma activa supone una decisión que tiene que ver con la percepción de sentirse capaz de tomarla. Las mujeres valoran a los profesionales de atención primaria porque su ayuda es importante para el proceso de salir. La forma pasiva implica no tomar una decisión por si mismas; frecuentemente es el agresor el que se va.

Ruiz Pérez y Plazaola ${ }^{18}$ comprobaron que el $63 \%$ de las mujeres que sufrían abuso llevaban a cabo algún tipo de acción para enfrentarse a la violencia del compañero íntimo. Las mujeres que se separaban de sus parejas eran mayoritariamente más jóvenes, con un menor número de hijos y más altos niveles de ingresos y educacionales, en comparación con las mujeres que sufrían abuso, que informaban a la policía o buscaban ayuda de los profesionales sanitarios o asociaciones de mujeres maltratadas. Factores independientes asociados con el hecho de presentar una respuesta a la violencia del compañero íntimo eran: estar separado o divorciado o viudo, tener apoyo social, haber experimentado violencia del compañero íntimo con frecuencia y haber sufrido abuso físico y psicológico (en comparación con haber sufrido abuso psicológico solamente). Las mujeres que experimentaban los tres tipos de abuso era más probable que respondieran a la violencia ${ }^{19}$.

Como señala Campbell ${ }^{20}$, las mujeres maltratadas están presentes en todos los ámbitos de atención sanitaria con diferentes problemas físicos y de salud mental, y diferentes características sociodemográficas. Las mujeres pueden presentarse en los servicios sanitarios antes de acudir a la justicia o a los servicios sociales, y si el abuso es identificado pueden recibir intervenciones que aumenten su seguridad y mejoren su salud.

El abuso es un factor de riesgo para muchos problemas de salud, pero su conocimiento es todavía insuficiente. Si el abuso se relaciona con factores como el tabaco, desnutrición, abuso de sustancias y estrés, las intervenciones sobre estos problemas no tendrán éxito sin enfrentar la violencia del compañero íntimo.

\section{BIBLIOGRAFÍA}

1. Observatorio de la Violencia de Género. Disponible en: http:// www.observatorioviolencia.org/ (con acceso el 3-2-2010).

2. Coker AL, Smith PH, Fadden MK. Intimate partner violence and disabilities among women attending Family Practice Clinics. J Womens Health (Larchmt). 2005; 14:829-38.

3. Bradley F, Smith M, Long J, O'Dowd T. Reported frequency of domestic violence: cross sectional survey of women attending general practice. BMJ. 2002; 324:271-4.

4. Ruiz-Pérez I, Plazaola-Castañoa J, Blanco-Prieto P, González-Barranco JM, Ayuso-Martín P, Montero-Piñar MI, y el Grupo de Estudio para la Violencia de Género. La violencia contra la mujer en la pareja. Un estudio en el ámbito de la atención primaria. Gac Sanit. 2006; 20:202-8.

5. Vives C, Álvarez-Dardet C, Caballero P. Violencia del compañero íntimo en España. Gac Sanit. 2003; 17:268-74.

6. Raya Ortega L, Ruiz Pérez J, Plazaola Castaño J, Brun López-Abisab S, Rueda Lozano D, García de Vinuesa L, et al. La violencia contra la mujer en la pareja como factor asociado a una mala salud física y psíquica. Aten Primaria. 2004; 34:117-27.

7. Aguar-Fernández M, Delgado-Sánchez A, Castellano-Arroyo M, Luna del Castillo JD. Prevalencia de malos tratos en mujer. Aten Primaria. 2006; 37:241-2.

8. Plazaola-Castaño J, Ruiz-Pérez I, Hernández-Torres E. Validación de la versión corta del Woman Abuse Screening Tool para su uso en atención primaria en España. Gac Sanit. 2008; 22: 415-20.

9. Ruiz-Pérez I, Plazaola-Castaño J, del Río-Lozano M. Physical health consequences of intimate partner violence in Spanish women. Eur J Public Health. 2007; 17:437-43.

10. Boyle A, Jones PB. The acceptability of routine inquiry about domestic violence towards women: a survey in three healthcare settings. Br J Gen Pract. 2006; 56:258-61.

11. García Torrecillas JM, Torío Durántez J, Lea Pereira MC, García Tirado MC, Aguilera Tejero R. Detección de violencia contra la mujer en la consulta del médico de familia. Aten Primaria. 2008; 40:455-61.

12. Richardson J, Coid J, Petruckevitch A, Chung WS, Moorey S, Feder G. Identifying domestic violence: cross sectional study in primary care. BMJ. 2002; 324:1-6.

13. Coid J, Petruckevitch A, Chung WS, Richardson J, Moorey S, Cotter S, Feder GS. Sexual violence against adult women primary care attenders in east London. $\mathrm{Br} J$ Gen Pract. 2003; 53:858-62.

14. Fernández MC. Violencia doméstica: ¿conocemos los efectos de la violencia sobre la salud de las víctimas? Aten Primaria. 2004; 34:125-7.

15. Hegarty K, Gunn J, Chondros P, Taft A. Physical and social predictors of partner abuse in women attending general practice: a cross-sectional study. Br J Gen Pract. 2008; 58: 484-7.

16. Sohal H, Eldridge S, Feder G. The sensitivity and specificity of four questions (HARK) to identify intimate partner violence: a diagnostic accuracy study in general practice. BMC Fam Pract. 2007; 8:49.

17. Muñoz Cobos F, Burgos Varo ML, Carrasco Rodríguez A, Martín Carretero ML, Río Ruiz J, Ortega Fraile I, Villalobos Bravo M. El torbellino de la violencia. Relatos biográficos de mujeres que sufren maltrato. Aten Primaria. 2009; 41: 493-500.

18. Rúiz-Pérez I, Plazaola-Castaño J. Intimate Partner Violence and Mental Health Consequences in Women Attending Family Practice in Spain. Psychosom Med. 2005; 67:791-7.

19. Rúiz-Pérez I, Plazaola-Castaño J, del Río-Lozano M, and the Gender Violence Study Group. How do women in Spain deal with an abusive relationship? J Epidemiol Community Health. 2006; 60:706-11.

20. Campbell JC. Health consequences of intimate partner violence. Lancet. 2002; 359:1331-6. 